\title{
Implicaciones del aumento del copago farmacéutico en España: una nueva privación material
}

\author{
Consequences of the Increase of the Pharmaceutical \\ Copayment in Spain: A New Material Deprivation
}

José Ángel Martínez-López (D) http://orcid.org/0000-0002-6871-7265

Universidad de Murcia, España,jaml@um.es

Gema Martínez-Gayo (D) https://orcid.org/0000-0003-1024-6429

Universidad Nacional de Educación a Distancia (UNED),España, gmartinez9@alumno.uned.es

\begin{abstract}
The law changes implemented by the Spanish government during the economic crisis started in 2007 have led to a restructuring of the pharmaceutical co-payment system. The aim of this research is to analyze what legislative changes have been introduced in health policy in Spain and how these reforms are influencing access to medicines. A methodological triangulation has been developed through the review of the specialized bibliography, analysis of the legislative changes as regards the pharmaceutical copayment implemented by the Spanish government as well as the most relevant databases with our object of study. Our research confirms that a social polarization is taking place in the Spanish population in relation to access to drugs, improved by a system of copayment that is not very progressive and does not take the needs of each social group into account.
\end{abstract}

Key words: health policy, social security for health, social expenditure, right to health and citizenship.

Resumen: Las reformas legislativas implementadas por el gobierno español durante la crisis económica iniciada en 2007 han supuesto una reestructuración del sistema de copago farmacéutico. El objetivo de esta investigación es analizar qué cambios legislativos se han introducido en la política de salud en España y cómo dichas reformas están influyendo en el acceso a los medicamentos. Para ello, se ha utilizado la triangulación metodológica para el desarrollo de la investigación, mediante la revisión de la bibliografía especializada, un examen de los cambios legislativos en materia de copago farmacéutico y un análisis de las bases de datos más relevantes

Recepción:

$02 / 05 / 18$

Aprobación: $14 / 03 / 19$ está produciendo una polarización social en la ciudadanía en relación con el acceso a los medicamentos, favorecida por un sistema de copago poco progresivo que no tiene en cuenta las necesidades de cada grupo social.

Palabras clave: política de salud, Seguridad Social en salud, gasto social, derecho a la salud, ciudadanía. 


\section{Introducción}

La protección a la salud es uno de los pilares que fundamentaron el Estado de bienestar en los países occidentales después del fin de la Segunda Guerra Mundial. Dicha protección tiene dos vertientes principales: atención en salud y farmacéutica. Tras las crisis económicas mundiales iniciadas en la década de 1970, se han incrementado los discursos en torno al cuestionamiento de la sostenibilidad del Estado de bienestar.

La última crisis económica y financiera que comenzó en 2007 en Estados Unidos ha afectado, tarde o temprano, al conjunto de países industrializados y ha supuesto una justificación para reestructurar el Estado de bienestar. Uno de sus principales baluartes en España está representado por la protección a la salud, caracterizándose desde sus inicios por su universalidad y gratuidad. Este sistema ha generado una sensación de seguridad en la ciudadanía junto con las pensiones de la Seguridad Social, el sistema educativo y, actualmente, con la protección social a las personas en situación de dependencia.

Algunas medidas adoptadas por el gobierno español para hacer frente a la crisis económica, se han centrado en la limitación de la universalidad en la atención a la salud a determinados colectivos y el incremento del copago farmacéutico al conjunto de la ciudadanía. Nos hallamos ante un posible cambio en el modelo de atención a la salud en España, que se caracteriza por una menor responsabilidad social, y puede consolidarse a través de los años, e incluso exportarse a otros países que se encuentren en una situación económica similar.

El objetivo de esta investigación es doble: 1) conocer las implicaciones de las reformas legislativas en materia farmacéutica en España; y 2) analizar sus posibles consecuencias en la ciudadanía. Dado que este fenómeno social es reciente, se realizó una aproximación al objeto de estudio mediante una combinación de estrategias metodológicas cuantitativas y cualitativas. Esta propuesta metodológica favorece el acercamiento a dicho problema social desde distintos planos, posibilita el debate de las implicaciones de los cambios normativos en materia de medicamentos y permite conocer cómo está afectando esta reconfiguración del sistema a la ciudadanía.

\section{Restricciones del copago farmacéutico y nuevos retos del Estado de bienestar en España}

El acceso a los medicamentos forma parte esencial de la atención a la salud, consagrando un derecho fundamental en la Carta Magna española 
(artículo 43). De esta manera, las medicinas no solo fomentan la recuperación o el tratamiento, sino son una parte indispensable de la protección a la salud. "El gasto en medicamentos es una de las principales líneas de gasto dentro de la salud pública de cualquier país que desee mantener un Estado de bienestar con unos servicios mínimos" (Lago et al., 2012: 23). Por tanto, el acceso a las medicinas forma parte de los principios de ciudadanía social (Marshall, 1950) y justicia equitativa y democrática (Rawls, 1991).

En los últimos años se han implementado diversas medidas para racionalizar el presupuesto en la atención a la salud desde un plano general. El presupuesto del Estado en medicamentos supone un coste importante dentro del Sistema Nacional de Salud, el cual se incrementa progresivamente por el envejecimiento de la población y la mayor necesidad de dar respuesta a pacientes crónicos polimedicados.

A la hora de analizar la evolución del gasto farmacéutico en un territorio, en este caso en España, nos enfrentamos a una serie de intereses contradictorios por parte de la industria farmacéutica. Por un lado, ocupa un lugar privilegiado para contribuir a la salud de la población e invierte grandes cantidades de dinero en innovación e investigación. Por otro, desde un plano mercantil, busca la maximización de beneficio como cualquier otro negocio, condicionando en ocasiones la integridad de investigaciones científicas. Por tanto, la industria farmacéutica no actúa al margen del mercado y a veces supedita las necesidades de la población a la rentabilidad económica (Páez, 2011).

La lógica del mercado farmacéutico no se dirige a las personas pobres ni trata de universalizar el acceso a los medicamentos, su desarrollo y prosperidad depende de las ventas, de la productividad y del potencial consumidor. Además, cabe tener en cuenta que la industria farmacéutica está controlada por compañías multinacionales, "cuyo poder financiero es enorme y al extender sus negocios globalmente lo hacen de maneras muy inequitativas o ventajosas" (Páez, 2016: 208).

La visión de enfermedad-curación mediante medicinas se encuadra en el modelo médico curativo clásico, que otorga a estos productos el protagonismo en la recuperación de la salud. Sin embargo, desde una visión crítica de la biomedicina, este modelo clásico dificulta las posibilidades de desarrollo de un modelo preventivo, que considere determinados factores individuales ligados al estilo de vida y la nutrición (Lifshitz, 2014), y cuestione el papel que juega la industria farmacéutica en el acceso a medicamentos de la ciudadanía.

El copago farmacéutico no es nuevo para los ciudadanos españoles, se configuró en 1966. La regulación actual se establece principalmente en el 
Real Decreto-Ley 16/2012, del 20 de abril (BOE, 2012a) y el Real Decreto Legislativo 1/2015, del 24 de julio (BOE, 2015). Estas normas jurídicas forman parte de las políticas de austeridad implementadas por el gobierno español, en el conjunto de sistemas de bienestar social a partir de la aprobación del Real Decreto-Ley 20/2012, del 13 de julio (BOE, 2012b). Todas ellas han redefinido el sistema de protección social y aumentado la probabilidad de empobrecimiento de los hogares. En relación con los cambios en materia de medicamentos, Freire (2014: 359) sugiere que "el RD Ley 16/2012 empeoró la situación de la cobertura poblacional de la sanidad pública dejando pendiente la única reforma de la misma que es coherente con los valores democráticos y de la sanidad pública, y con la experiencia de los países más ejemplares: proclamar en positivo la cobertura sanitaria universal e igual para todos".

Las implicaciones de los cambios normativos en la protección farmacéutica en España están siendo abordadas por investigadores como Garrido (2014), Cantero (2014), Jiménez-Martín y Viola (2014, 2016), desde perspectivas heterogéneas. El Estado interviene regulando el precio de los medicamentos, para que no existan conflictos de intereses y la salud no esté supeditada al interés económico. En los países desarrollados "se regula el acceso, la producción, la promoción y el precio de los medicamentos porque el mercado no se basta” (Ortún, 2008: 112).

En una sociedad global caracterizada por un aumento de la precarización de las condiciones laborales y el desempleo, el acceso a la prestación farmacéutica se podría estar convirtiendo en un bien privilegiado, que no se pueden permitir todas las personas. "Desde la perspectiva de la equidad, dichos copagos pueden afectar negativamente a algunos segmentos de la población, especialmente a los enfermos crónicos y los de renta más baja” (Saludas, 2013: 43).

Las crecientes dificultades económicas de la ciudadanía, unidas a la incidencia del copago farmacéutico, han propiciado que "el número de hogares que han dejado de comprar medicinas, seguir dietas o tratamientos por problemas económicos sean ahora casi el triple en términos de población, alcanzando al 15,5\%" (Foessa, 2014: 162). Además, existen evidencias de que el copago farmacéutico no afecta a todos por igual, sino que repercute negativamente en la salud de los más desfavorecidos (Aron-Dine et al., 2013). Un estudio realizado en 2005 observó que "los pacientes responden de manera diferente a un aumento del copago farmacéutico para los medicamentos recetados dependiendo de la afección tratada, el aumento absoluto de los precios y la disponibilidad de alternativas de tratamiento" 
(Landsman et al., 2005: 627). Por tanto, hay una interconexión de factores en el uso de medicamentos, y cualquier análisis debe considerar el conjunto de variables.

Una gran parte de las medidas destinadas a la reducción del gasto farmacéutico se está orientando al incremento del copago y la exclusión de medicinas de la cartera de servicios. Sin embargo, existen otras alternativas, como crear "un nuevo marco regulador, más estable y transparente, que explicite y jerarquice los criterios considerados, y que esté apoyado en estudios de evaluación económica llevados a cabo por un órgano independiente del gobierno" (Cabiedes, 2013: 412). Es decir, centrar las medidas en el aumento del copago sanitario y la exclusión de medicamentos del catálogo farmacéutico no parece ser una solución factible ni justa a largo plazo.

Otras propuestas en la gestión se dirigen a reorientar la prescripción farmacéutica hacia un uso racional. "Este abordaje puede ser una oportunidad para reducir el despilfarro y mejorar la eficiencia del Sistema Nacional de Salud, pero, hoy por hoy, es sobre todo una oportunidad para mejorar la salud y el bienestar de nuestros pacientes" (Sanfélix-Gimeno et al., 2011: 66).

En España ya existe un copago sanitario por medio de los impuestos: de forma progresiva a través del Impuesto sobre la Renta de las Personas Físicas (IRPF), o proporcional, a través del Impuesto sobre el Valor Añadido. Pero, el acceso a los medicamentos está condicionado al copago directo que realizan los pacientes al adquirir cada uno. El incremento de este copago puede ocasionar la privación en el acceso al mismo. De hecho, hay investigaciones que evidencian los factores que interfieren en la decisión de los pacientes de comprar o no medicinas, tras ser atendidos en los servicios de urgencias, al ser el coste de ellas una de las variables (Rodríguez y Puig-Junoy, 2013).

En esta línea, otros estudios (Saludas, 2013; Cortès-Franch y González, 2014) muestran que el copago farmacéutico desincentiva el consumo de aquellos medicamentos innecesarios o sustituibles y que las mismas personas racionalizan su consumo. De este modo, se produce una autovaloración de la necesidad de adquirirlos, en virtud de la capacidad económica, quedando en segundo lugar la mejora que pueden producir en la salud. Las distintas reformas legislativas en los sistemas de salud suponen una reducción de la cobertura sanitaria y amplían el espacio de desigualdad entre la ciudadanía (Foessa, 2014).

Puig-Junoy (2007) aboga por el establecimiento de copagos diferenciales: 1) un copago reducido para medicamentos esenciales, 2) otro más elevado para los preferenciales y 3 ) un tercero más elevado para los considerados no preferenciales y menos eficientes. "Una de las ventajas adicionales 
de los copagos diferenciales es que fomenta la competencia entre sustitutos terapéuticos para ser incluidos en los grupos de copago más reducido y facilita, al mismo tiempo, la negociación descentralizada de precios (descuento o retorno) entre financiadores e industria farmacéutica" (Puig-Junoy, 2007: 157-158).

Tras las reformas legislativas existe el riesgo de que el copago farmacéutico termine convirtiéndose en un “'impuesto sobre la enfermedad' y que no sirva para cambiar la tendencia al aumento de la prescripción [...]. La crisis parece que está dificultando el acceso a los servicios sanitarios de los grupos socioeconómicos más desfavorecidos, incluyendo a las personas en situación de inmigración irregular" (Cortès-Franch y González, 2014: 3). Además, las modificaciones en la protección farmacéutica han sido muy severas y poco equitativas, poniendo en evidencia la desigualdad de oportunidades, así como la conformación de nuevas barreras para alcanzar la ciudadanía social. Los cambios normativos relativos a la protección a la salud se rigen por la ley del inversamente proporcional: "cuanta más necesidad de atención sanitaria tiene una persona o un grupo social, menor es su acceso real a una atención sanitaria de calidad" (Navarro et al., 2016: 1271).

La privación material en el uso de medicamentos es un ejemplo del aumento de las tendencias de pobreza y de la desigualdad, como consecuencia del desarrollo de políticas neoliberales que afectan de forma más intensa a las personas en situación de pobreza. Recientemente, Oxfam (2016: 40) propuso como mecanismo para reducir la desigualdad en este ámbito "modificar el sistema mundial de investigación y desarrollo $(\mathrm{I}+\mathrm{D})$ y de fijación de los precios de los medicamentos para garantizar el acceso de todas las personas a medicamentos adecuados y asequibles". El actual escenario económico y social puede estar propiciando nuevos perfiles de pobreza, que se podrían asociar con ciertos niveles de privación material, entre ellos la privación a la salud por la imposibilidad de consumir medicinas.

\section{Metodología}

La aproximación al objeto de estudio se ha realizado desde una triangulación metodológica. Partimos de la premisa de que en las ciencias sociales no existe un método mejor que otro, y que la combinación de ellos, mediante la pluralidad de enfoques, permite un mejor acercamiento a la realidad social. La triangulación metodológica, a partir de la combinación de datos, metodologías o teorías (Denzin, 1978), posibilita "adquirir un conocimiento más amplio y profundo del objeto de análisis” (Cantor, 2002: 
59). La triangulación tiene importantes ventajas, ya que mejora la confianza en los resultados y estimula la invención o introducción de nuevos métodos (Samaja, 2018).

En esta investigación se aborda un fenómeno nuevo en España, que precisa ser estudiado desde diferentes perspectivas, para tener una concepción integral del mismo. Dado que tratamos de conocer las implicaciones de los cambios legislativos en la población española, si nos ciñésemos a un solo enfoque, la información obtenida del problema social sería sesgada y parcial. Por ello, se utilizó una triangulación metodológica a partir del análisis teórico y de la combinación de métodos de análisis de datos, usando técnicas cualitativas y cuantitativas en etapas secuenciales.

En un primer momento se hizo una revisión teórica sobre el estado de la cuestión, prestando especial atención al concepto de salud como derecho, al papel que juega el Estado de bienestar en la regulación de los medicamentos, y a los estudios más recientes sobre la privación en el acceso a los mismos en el actual contexto económico y social en España.

Posteriormente, desde una vertiente cualitativa, se han estudiado las distintas reformas legislativas en relación con el acceso a medicinas que tuvieron lugar en España en el último lustro, desde un punto comparativo con las iniciadas en el año 1966. Para ello, se consultaron las novedades legislativas en dicha materia, publicadas en el Boletín Oficial del Estado (BOE). Los principales elementos de análisis son: 1) número de estratos establecidos en el copago sanitario, 2) rangos de ingresos de cada estrato, 3) porcentaje de participación y límites máximos de cada estrato, 4) exclusión de medicamentos protegidos por el Sistema Nacional de Salud y 5) protección social a colectivos especialmente vulnerables (personas pensionistas con rentas bajas y desempleados, sobre todo).

Finalmente, y desde una vertiente cuantitativa, se examinaron las fuentes de datos más destacadas respecto al presente objeto de estudio, en particular el Barómetro Sanitario y las publicaciones de la EAE Business School en esta materia. El Barómetro Sanitario es un estudio de opinión anual del Ministerio de Sanidad, Servicios Sociales e Igualdad y del Centro de Investigaciones Sociológicas; ayuda a conocer, entre otros temas, el grado de satisfacción de la ciudadanía sobre los servicios sanitarios públicos a lo largo del tiempo. Por su parte, "El gasto farmacéutico en España en 2016" es un informe de la EAE Business School (2016), orientado al estudio del gasto farmacéutico público y privado, incidiendo en la evolución del mismo en las diferentes regiones españolas, y en el esfuerzo realizado por las familias para acceder a estos productos. Centramos nuestro análisis de datos en tres elementos clave: 
1) la inversión en medicamentos a partir de la introducción de las reformas legislativas en España, 2) el gasto en medicinas por habitante, y 3) el perfil y porcentaje de la población que no puede acceder a medicamentos prescritos por encontrarse en situación de vulnerabilidad social.

El contexto temporal analizado ha sido amplio, sobre todo en el caso de la legislación en materia farmacológica, para comprender los cambios producidos, considerando especialmente el inicio de la crisis económica (2007) y la introducción de las medidas de austeridad que afectaron a este sector en el año 2012.

\section{Recorrido histórico de la participación en el copago farmacéutico ${ }^{1}$}

Los referentes legislativos más importantes en la protección a la salud los encontramos en el artículo 43 de la Constitución Española y en la Ley General de Sanidad, aprobada en 1986 (BOE, 1986), con los cuales se estableció el Sistema Nacional de Salud en España. Veinte años después se aprobó la Ley 29/2006, del 26 de julio de garantías y uso racional de los medicamentos y productos sanitarios (BOE, 2006a). Sin embargo, los antecedentes reguladores de la prestación farmacéutica se extienden aún más atrás en el tiempo.

El primer vestigio del copago farmacéutico en España se halla en el Decreto 3157/1966, del 23 de diciembre (BOE, 1966), por el cual se regula la dispensación de especialidades farmacéuticas en el Régimen General de la Seguridad Social. En su artículo 3 se recogía de forma expresa que la asistencia farmacéutica sería gratuita cuando se administrara en instituciones sanitarias públicas o concertadas o derivasen de accidentes de trabajo o enfermedades profesionales. Además, en ese mismo artículo se establecía un copago para los medicamentos dependiendo del precio de estos, según fuese inferior, o igual o superior a 30 pesetas. En todo caso, el copago mínimo de la medicina estaba establecido en cinco pesetas, es decir, un mínimo de 16,6\%. El porcentaje de copago se podía incrementar en una peseta más por cada decena del medicamento sin que en ningún caso excediera de 50 pesetas. Cabe destacar que no se fijaba gratuidad o descuento para ningún colectivo, ni siquiera para los pensionistas.

1 En el análisis normativo de este apartado especificamos que el orden jerárquico legislativo en España es el siguiente: Constitución Española, Ley Orgánica, Ley (Ordinaria), Real Decreto Ley, Real Decreto Legislativo, reglamentos, resoluciones, así como otras normas de rango inferior. Antes de la aprobación de la Constitución Española de 1978, las normas jurídicas adoptaban principalmente la forma de Ley, Decreto y Orden. 
Posteriormente, se aprobó el Real Decreto 945/1978, del 14 de abril (BOE, 1978) por el cual se da una nueva regulación a la aportación del beneficiario de la Seguridad Social en la dispensación de las especialidades farmacéuticas. Esta norma jurídica incorpora importantes novedades. Por un lado, reconoce la exención del copago para las personas pensionistas de la Seguridad Social y, por primera vez, a aquellos trabajadores que se encontraran en situación de invalidez provisional derivada de enfermedad común o accidente no laboral. Además, esta norma jurídica incluía un anexo de medicinas (mayoritariamente para patologías crónicas), que se seguirán rigiendo por la norma de 1966. Al resto se le aplicaría un copago del 30\% de la medicina.

Dos años después se legisló el Real Decreto 1605/1980, del 31 de julio (BOE, 1980), por el cual se modifican los tipos de cotización para desempleo, Seguridad Social y Fondo de Garantía Salarial, y se revisa el porcentaje de participación de los beneficiarios en el precio de determinados medicamentos. Este Real Decreto incorpora una importante modificación: a partir de este momento la participación en el sistema farmacéutico para el conjunto de personas no excluidas en el copago ascendería a $40 \%$.

En 2006 se aprobó la Ley 29/2006, del 26 de julio (BOE, 2006a) de garantías y uso racional de los medicamentos y productos sanitarios que posteriormente se desarrolló en el Real Decreto 1030/2006, del 15 de septiembre (BOE, 2006b) por el cual se establece la cartera de servicios comunes del Sistema Nacional de Salud y el procedimiento para su actualización. Las modificaciones más importantes que incorpora esta norma jurídica son: en primer lugar, la exclusión de los siguientes medicamentos en la protección a la Seguridad Social: a) los productos de utilización cosmética, dietéticos, aguas minerales, elíxires, dentífricos y otros productos similares; b) los medicamentos calificados como publicitarios, c) los medicamentos adscritos a los grupos o subgrupos terapéuticos excluidos de la financiación por la normativa vigente; d) los medicamentos homeopáticos; e) los efectos y accesorios de los que se realice publicidad dirigida al público en general. En segundo lugar, se establecen los siguientes criterios para el copago. Por un lado, hay un sistema general denominado "aportación normal", que alcanza el $40 \%$ del coste del producto, en línea con la normativa anterior. Por el otro, se establece otra modalidad: "aportación reducida”, donde los pacientes tendrían que abonar únicamente el $10 \%$ del producto sin que en 
ningún caso excediera de 2,64€. ${ }^{2}$ Además, se amplía el grupo de personas, tratamientos y productos exentos de estas aportaciones: 1) pensionistas y colectivos asimilados, afectados de síndrome tóxico y personas con discapacidad en los supuestos contemplados en su normativa específica; 2) tratamientos derivados de accidentes de trabajo y enfermedad profesional; y c) productos cuya dispensación al usuario se realice en los centros o servicios asistenciales sanitarios.

Una vez que iniciaron la crisis económica y las políticas de restricción del gasto, se aprobó la norma jurídica de mayor alcance en la protección a la asistencia farmacológica, el Real Decreto-Ley 16/2012, del 20 de abril (BOE, 2012a), de medidas urgentes para garantizar la sostenibilidad del Sistema Nacional de Salud y mejorar la calidad y seguridad de sus prestaciones. Este Real Decreto-Ley incorpora modificaciones importantes en la Ley 29/2006, del 26 de julio (BOE, 2006a) de garantías y uso racional de los medicamentos y productos sanitarios, especialmente en el artículo 94 bis, que vincula el copago farmacéutico con la capacidad económica. Como regla general, los usuarios pagarán a partir de este momento el $60 \%$ del precio de venta al público (PVP) de la medicina siempre y cuando tengan una renta igual o superior a $100.000 €$ en su declaración del Impuesto sobre la Renta de las Personas Físicas. Para quienes tengan ingresos de entre 18.000 y $100.000 €$, su aportación será el 50\% del PVP.

Por otro lado, las personas que se encuentren aseguradas en activo y tengan unos ingresos inferiores a $18.000 €$ deberán aportar 40\% del PVP. Finalmente, quienes son pensionistas de la Seguridad Social pagarán un $10 \%$ del PVP, siempre y cuando no tengan unos ingresos iguales o superiores a $100.000 €$.

Esta norma jurídica recoge una serie de garantías para las personas pensionistas con tratamientos de larga duración, con el propósito de facilitar el acceso a los medicamentos. Para ello, se estableció una serie de límites en el copago, fijando unos topes máximos sobre los cuales sería gratuita la adquisición de medicinas. En el caso de pensionistas que tomen medicamentos pertenecientes a los grupos $\mathrm{ATC}^{3}$ y de aportación reducida, abonarán un 10\% del precio del producto con un máximo, para el año 2012, de 2,64€.

2 Corresponde abonar este tipo de aportación en los siguientes supuestos: a) medicamentos para el tratamiento de enfermedades crónicas o graves, clasificados en los grupos o subgrupos terapéuticos recogidos en la normativa vigente y de acuerdo con las condiciones establecidas. b) Efectos y accesorios pertenecientes a los grupos reglamentariamente establecidos. c) Medicamentos que proporciona el Sistema Nacional de Salud, por medio de la receta oficial a los enfermos de Sida.

3 Listado codificado de sustancias organizadas por principio activo y medicamentos según el sistema u órgano afectado. 
Para los pensionistas con ingresos inferiores a $18.000 €$, el límite es de $8 €$. En el caso de quienes tengan ingresos iguales o superiores a $18.000 €$ y hasta $100.000 €$, el límite por abonar será de $18 €$. Por último, para los pensionistas con ingresos iguales o superiores a $100.000 €$, el importe máximo por abonar será de $60 €$. Estas cantidades se han ido revalorizando cada año de acuerdo con el índice de precios al consumo (IPC). Además, también se establecía una serie de exenciones para determinados colectivos como por ejemplo: 1) afectados de síndrome tóxico y personas con discapacidad en los supuestos contemplados en su normativa específica; 2) personas perceptoras de rentas de integración social; 3 ) personas perceptoras de pensiones no contributivas; 4) parados que han perdido el derecho a percibir el subsidio de desempleo en tanto dure su situación; y 5) los tratamientos derivados de accidente de trabajo y enfermedad profesional.

Esta norma jurídica modificó también el Real Decreto 1718/2010, del 17 de diciembre (BOE, 2010) sobre receta médica y órdenes de dispensación en relación con los códigos para establecer el copago farmacéutico. Estos quedarían recogidos de la siguiente manera: a) Código TSI 001 para los usuarios exentos de aportación; b) Código TSI 002 para los usuarios con aportación reducida de un 10\%; c) Código TSI 003 para los usuarios con aportación de un 40\%; d) Código TSI 004 para los usuarios con aportación de un 50\%; e) Código TSI 005 para los usuarios con aportación de un $60 \%$; f) ATEP para las recetas de accidentes de trabajo o enfermedad profesional; g) NOFIN para las recetas de medicamentos y productos sanitarios no financiados. Los cambios más relevantes introducidos respecto a la prestación farmacéutica desde su primera regulación hasta la actualidad aparecen en la Tabla I. ${ }^{4}$

Tres años más tarde, se legisló el Real Decreto Legislativo 1/2015, del 24 de julio (BOE, 2015) por el cual se aprueba el texto refundido de la Ley de garantías y uso racional de los medicamentos y productos sanitarios. Esta norma no recoge cambios sustanciales en relación con el copago, salvo una actualización del coste máximo de la participación en el sistema, de acuerdo con la capacidad económica de los pacientes. El sistema de copago español quedó establecido, tras estas reformas, tal y como aparece en la Tabla II.

Además, cabe señalar un elemento muy destacado, que, aunque no se contempla dentro del copago farmacéutico, está conectado con él: la exclusión de medicamentos del sistema sanitario. Este hecho tuvo lugar con la aprobación de la Resolución del 2 de agosto de 2012 (BOE, 2012c), de la Dirección General de Cartera Básica de Servicios del Sistema Nacional de

4 Todos las tablas y gráficos se encuentran en el Anexo, al final del presente artículo. 
Salud y Farmacia, por la cual se procede a la actualización de la lista de medicamentos que quedan excluidos de la prestación farmacéutica en el Sistema Nacional de Salud. Un total de 417 dejaron de tener cobertura por el sistema de Seguridad Social, suponiendo un ahorro de 450 millones de euros (Ministerio de Sanidad, Política Social e Igualdad, 2012).

Dentro de estas medicinas se podían encontrar productos íntimamente relacionados con dolencias o problemas médicos comunes como antiácidos, laxantes, antidiarreicos y aquellos destinados a los problemas musculares, para la tos, alergia o afecciones neumológicas. Por tanto, se produjo un incremento del copago farmacéutico por acción, al aumentar la participación de los pacientes en el costo de estos productos, así como por omisión, al excluir de la cobertura sanitaria una amalgama de medicamentos de uso cotidiano y de carácter crónico. ${ }^{5}$

Si bien se establece un sistema estratificado, carece de las franjas necesarias para considerarlo equitativo. "Parece oportuno que se estratifique más la población más allá de los umbrales de renta actualmente reconocidos (menos de 18.000 euros, de 18.000 a 100.000 euros, más de 100.000 euros), sería oportuno tomar en consideración, más allá del nivel de renta, también la riqueza patrimonial de los distintos individuos" (Lucas, 2016: 67).

Resulta difícil concretar el impacto total que tuvo para la ciudadanía las modificaciones legislativas en materia de medicamentos. Lo que sí es obvio es que supuso un cambio del modelo de protección existente hasta ese momento, devaluando los derechos adquiridos, aumentando la dificultad de obtener un recurso básico de primera necesidad, e incrementando la desigualdad social en las personas en situación de pobreza o insertas en procesos de exclusión social.

\section{La evolución del gasto farmacéutico en España}

Si nos aproximamos a los diferentes modelos de financiación de la prestación farmacéutica podemos observar su diversidad: desde los que representan una fuerte responsabilidad pública y social en el acceso a los medicamentos, hasta los que se apoyan en la familia como principal agente proveedor de estos. Dado el actual contexto económico mundial, no resulta extraño que algunos países - Grecia, Irlanda, Italia, Portugal, entre otros- hayan realizado ajustes en gastos sanitarios, y dentro de ellos, en los farmacéuticos, con el fin de reducir sus niveles de déficit, a través de la introducción del copago

5 Algunos de estos medicamentos son habituales en nuestros botiquines como por ejemplo: Almax, Fortasec, Fastum, Calmatel, Movilisin, Zenavan, Rinomax, Mucosan o Pectox. 
farmacéutico o la reducción del gasto medio por receta, entre otros (EAE Business School, 2016).

No obstante, lo que para unos supone una medida para mejorar la eficiencia del gasto público y hacerlo sostenible, es para otros una manera de debilitar las prestaciones del Estado de bienestar, que acaba por empeorar la situación de quienes son más vulnerables y no pueden acceder, o lo hacen con dificultad, a los fármacos prescritos.

España presentaba en 2014 un gasto en medicamentos, material y otros productos farmacéuticos que suponía 1,63\% del Producto Interior Bruto (PIB) (EAE Business School, 2016). Dentro de este porcentaje, el gasto público y el gasto privado han experimentado una tendencia opuesta en los últimos nueve años. El gasto público se ha reducido especialmente tras 2009, mientras que el gasto privado se ha incrementado en esos mismos productos. La variación del gasto total en medicamentos y otros productos farmacéuticos entre 2009 y 2014 se saldó para España con una reducción de $0,77 \%$. No obstante, la variación interanual respecto al año 2013 arroja una reducción superior al 3,4\% (EAE Business School, 2016).

Una vez establecida la situación española en cuanto a gasto farmacéutico, resulta significativo estudiar la evolución del gasto público y de los hogares en los últimos años. En este periodo, como se ha mencionado antes, se están viviendo situaciones de crisis económica y financiera, que han llevado a muchos territorios a contraer el gasto en determinadas partidas presupuestarias. Esta forma de gestión supone un cuestionamiento a los derechos adquiridos y fomenta la desigualdad social, ya que el Estado de bienestar se debilita. "A nivel micro la enfermedad crea la pobreza y esa pobreza crea más enfermedad [...] A nivel macro el círculo vicioso es más importante todavía; el incremento de los problemas sanitarios demanda un incremento de esos recursos sanitarios" (Navarro et al., 2016: 1271). Obviar estas realidades supone ser ciegos a cómo las restricciones presupuestarias en el acceso a los medicamentos son un componente generador de desigualdades sociales.

Tanto el gasto público, como el personal realizado por los hogares, en medicinas han experimentado en este periodo una evolución contrapuesta. Mientras que el esfuerzo personal realizado por los hogares en este tipo de bienes no ha dejado de crecer desde 2012 y hasta 2015, el gasto público descendió sin cesar entre 2010 y 2013, para incrementarse ligeramente en los dos últimos años analizados, pero con cifras aún lejanas a las registradas en 2007. El descenso experimentado para la totalidad del periodo excede los 50 euros. 
Por su parte, los hogares han tenido que aportar más para poder acceder a los medicamentos prescritos, suponiendo un aumento de casi el 16\% en estos ocho años. En el último trienio se ha podido observar cómo las cifras de gasto de unos y otros se han aproximado (EAE Business School, 2016) (véase Gráfico 1).

Sin embargo, no todas las personas han visto incrementarse de igual forma estas facturas farmacéuticas. Los datos proporcionados por el informe "El gasto farmacéutico en España en 2016" (EAE Business School, 2016) indican que son las mujeres, con independencia de su edad, quienes soportan un mayor gasto en este tipo de productos, en especial en 2013 tras la entrada en vigor de la "Reforma de Salud". Las personas mayores de 65 años son las que más gastan en esta clase de productos; además, los hombres que superan esta edad son quienes han visto en mayor medida incrementarse este gasto farmacéutico en estos ocho años, prácticamente en 39\%. El análisis del gasto medio por tipo de hogar nos desvela que el mayor aumento en esos años correspondió en aquellos hogares de una sola persona de 65 o más años, llegando a superar el incremento en más de 54\%, lo cual les hizo asumir un coste farmacéutico que superó los 365 euros anuales en 2015 (EAE Business School, 2016).

Todo ello ha supuesto un incremento de la inversión de la ciudadanía en productos farmacéuticos y ha influido en el porcentaje de personas que en los últimos 12 meses ha dejado de tomar algún medicamento recetado por la sanidad pública al no poder permitírselo por sus recursos económicos. Los datos analizados procedentes de los Barómetros Sanitarios, elaborados por el Ministerio de Sanidad, Servicios Sociales e Igualdad (2014-2017) español, nos permiten observar que tras la entrada en vigor de la llamada "Reforma de Salud" se registró un porcentaje de personas que no puede acceder a los medicamentos prescritos de poco más del $5 \%$, dato que se verá reducido en los dos años posteriores para volver a incrementarse en 2016 (véase Gráfico 2).

Pero el incremento de este copago sanitario no ha afectado de manera homogénea a toda la ciudadanía; se han encontrado grupos más vulnerables que otros ante la implementación del mismo. En 2016 son las mujeres, los jóvenes (18-24 años) y aquellas personas de entre 45 y 54 años quienes concentran este mayor riesgo (Ministerio de Sanidad, Servicios Sociales e Igualdad, 2017). Si tradicionalmente estos grupos sociales se caracterizaban por la carencia en el pago de recibos de suministros (luz, agua, impuestos municipales) o relacionados con la vivienda, ahora están aumentando aquellos que se ven incapaces de autoproveerse de ciertos niveles de calidad en su salud. Y a diferencia de lo que pudiera pensarse, las personas mayores no tienen por qué 
ser quienes se encuentren en una peor posición relativa (por sus escasas cuantías en las pensiones), ya que donde más han aumentado las tasas de pobreza y exclusión social es en la población activa (Instituto Nacional de Estadística, 2017). Las necesidades no cubiertas relacionadas con el consumo de medicamentos no dejan de ser una privación sanitaria, poniendo en entredicho la ciudadanía social y uno de los baluartes del Estado de bienestar en España.

\section{Conclusiones y discusión}

Uno de los rasgos más distintivos a la hora de analizar los Estados de bienestar es el grado de protección social que este ofrece a su ciudadanía. Junto con la educación, los servicios sociales y las pensiones, la salud supone uno de los pilares fundamentales de ese sistema de protección en España. A pesar de esta importancia y del acceso universal que tradicionalmente ha definido a estos servicios, parece que nos encontramos en un momento de "cambio de modelo". Este se caracterizaría por una menor participación social y un aumento del individualismo en la protección social. Tanto las características propias del Estado de bienestar español -caracterizado por familismo intenso-, como las reformas introducidas tras el inicio de la crisis económica y mundial, han debilitado la extensión del modelo existente.

En el ámbito concreto de la salud, las prestaciones farmacéuticas se han visto limitadas al entrar en vigor varias normas jurídicas, sobre todo, a partir de la aprobación del Real Decreto Ley 16/2012 (BOE, 2012a). Las implicaciones en la ciudadanía han sido numerosas, pero cabe destacar dos. En primer lugar, se ha producido un incremento de la cuantía del copago farmacéutico por parte de la ciudadanía de manera poco progresiva. En segundo, se ha excluido un elevado número de medicamentos de la cobertura del Sistema Nacional de Salud, suponiendo un aumento del gasto farmacéutico para la ciudadanía. Por tanto, esta ha tenido que enfrentar dicho incremento en condiciones de inequidad, lo cual ha conducido al crecimiento de la desigualdad social y menoscabo de los derechos adquiridos. Dichas medidas supusieron un ahorro en las arcas públicas, pero también generaron críticas, dado que existen otras propuestas para racionalizar el gasto que no recaen de una forma tan directa sobre el bienestar de la ciudadanía. Todo ello se tradujo en una disminución de la aportación al gasto farmacéutico de acuerdo con el PIB en España.

El incremento de este gasto en los hogares no se ha repartido de manera homogénea. Las personas polimedicadas y en especial las mayores de 65 años son quienes presentan una factura farmacológica más elevada. Sin embargo, 
no han sido estas personas las que soportan las mayores dificultades de acceso a su medicación. Según el Barómetro Sanitario 2016 son las personas más jóvenes y las de entre 45 y 54 años quienes, en mayor medida, afirman no poder acceder a la medicación prescrita por su facultativo debido a problemas económicos. Este hecho tiene mayor incidencia en las mujeres que en los hombres españoles, siendo ellas quienes también afrontan un mayor gasto por este concepto (Ministerio de Sanidad, Servicios Sociales e Igualdad, 2017).

El aumento del copago farmacéutico se está realizando en un momento en el que el resto de prestaciones sociales derivadas del sistema de protección social se han reducido y existen unas cifras elevadas de desempleo. Como consecuencia, se están incrementando los niveles de desigualdad y pobreza, y favoreciendo el desarrollo de una sociedad cada vez más polarizada que no garantiza la calidad de vida de su ciudadanía.

Además, la participación en el gasto farmacéutico recae especialmente en personas con enfermedades crónicas, polimedicadas, con rentas bajas y que podrían tener que dejar de acceder a dichos fármacos. Si bien las medidas de participación de los beneficiarios en el coste de estos productos podrían traer racionalidad y equilibrio a las arcas públicas, el efecto disuasorio sobre este bien de primera necesidad, dentro de un marco socioeconómico de incremento de las tendencias de pobreza y exclusión social en España, supone una barrera para las personas que aspiran a gozar de estándares mínimos de calidad en materia de salud. Y lo que es más importante, no se pone el énfasis en la necesidad de abordar un cambio en el modelo de gestión del copago farmacéutico.

\section{Referencias}

Aron-Dine et al. (2013), “The RAND health insurance experiment, three decades later", en The Journal of Economic Perspectives, núm. 27, vol. 1, Estados Unidos: American Economic Association.

BOE [Boletín Oficial del Estado] (1966), Decreto 3157/1966, de 23 de diciembre, por el que se regula la dispensación de especialidades farmacéuticas en el Régimen General de la Seguridad Social, España: BOE. Disponible en: http://www.boe.es/buscar/doc. php?id=BOE-A-1966-21115 [1 de julio de 2017].

BOE [Boletín Oficial del Estado] (1978), Real Decreto 945/1978, de 14 de abril por el que se da nueva regulación a la aportación del beneficiario de la Seguridad Social en la dispensación de las especialidades farmacéuticas, España: BOE. Disponible en: http:// www.boe.es/buscar/doc.php?id=BOE-A-1978-13676 [1 de julio de 2017].

BOE [Boletín Oficial del Estado] (1980), Real Decreto 1605/1980, de 31 de julio, por el que se modifican los tipos de cotización para desempleo, Seguridad Social y Fondo de Garantía Salarial y se revisa el porcentaje de participación de los beneficiarios en el precio 
de determinados medicamentos, España: BOE. Disponible en: http://www.boe.es/ buscar/doc.php?id=BOE-A-1980-16601 [1 de julio de 2017].

BOE [Boletín Oficial del Estado] (1986), Ley 14/1986, de 25 de abril, General de sanidad, España: BOE. Disponible en: https://www.boe.es/buscar/act. php?id=BOE-A-1986-10499 [ 1 de julio de 2017].

BOE [Boletín Oficial del Estado] (2006a), Ley 29/2006, de 26 de julio, de garantías y uso racional de los medicamentos y productos sanitarios, España: BOE. Disponible en: http://www.boe.es/buscar/act.php?id=BOE-A-2006-13554 [15 de julio de 2017].

BOE [Boletín Oficial del Estado] (2006b), Real Decreto 1030/2006, de 15 de septiembre, por el que se establece la cartera de servicios comunes del Sistema Nacional de Salud y el procedimiento para su actualización, España: BOE. Disponible en: http://www.boe.es/ buscar/act.php?id=BOE-A-2006-16212 [1 de agosto de 2017].

BOE [Boletín Oficial del Estado] (2010), Real Decreto 1718/2010, de 17 de diciembre, sobre receta médica y órdenes de dispensación, España: BOE. Disponible en: http://www.boe. es/buscar/act.php?id=BOE-A-2011-1013 [15 de agosto de 2017].

BOE [Boletín Oficial del Estado] (2012a), Real Decreto-ley 16/2012, de 20 de abril, de medidas urgentes para garantizar la sostenibilidad del Sistema Nacional de Salud y mejorar la calidad y seguridad de sus prestaciones, España: BOE. Disponible en: http:// www.boe.es/buscar/act.php?id=BOE-A-2012-5403 [16 de agosto de 2017].

BOE [Boletín Oficial del Estado] (2012b), RealDecreto-ley 20/2012, de 13 dejulio, demedidas para garantizar la estabilidad presupuestaria y de fomento de la competitividad, España: BOE. Disponible en: http://www.boe.es/buscar/doc.php?id=BOE-A-2012-9364 [16 de agosto de 2017].

BOE [Boletín Oficial del Estado] (2012c), Resolución de 2 de agosto de 2012, de la Dirección General de Cartera Básica de Servicios del Sistema Nacional de Salud y Farmacia, por la que se procede a la actualización de la lista de medicamentos que quedan excluidos de la prestación farmacéutica en el Sistema Nacional de Salud, España: BOE. Disponible en: http://www.boe.es/diario_boe/txt.php?id=BOE-A-2012-10952 [07 de septiembre de 2017].

BOE [Boletín Oficial del Estado] (2015), Real Decreto Legislativo 1/2015, de 24 de julio, por el que se aprueba el texto refundido de la Ley de garantías y uso racional de los medicamentos y productos sanitarios, España: BOE. Disponible en: http://www.boe. es/diario_boe/txt.php?id=BOE-A-2015-8343 [16 de agosto de 2017].

Cabiedes, Laura (2013), "Nuevas perspectivas sobre el precio de los medicamentos: El caso español”, en Estudios de Economía Aplicada, núm. 31, vol. 2, España: Universidad de Valladolid.

Cantero, Josefa (2014), "Constitución y derecho a la protección de la salud", en Revista CESCO de Derecho de Consumo, núm. 8, España: Universidad de Castilla-La Mancha.

Cantor, Guillermo (2002), "La triangulación metodológica en las ciencias sociales", en Cinta de Moebio, núm. 13, Chile: Universidad de Chile.

Cortès-Franch, Inma y González, Beatriz (2014), "Crisis económica-financiera y salud en España. Evidencia y perspectivas. Informe SESPAS 2014”, en Gaceta Sanitaria, núm. 28 (S1), España: Sociedad Española de Salud Pública y Administración Sanitaria (SESPAS).

Denzin, Norman (1978), The research act. A theoretical introduction to sociological methods, Estados Unidos: Mc Graw Hill. 
EAE Business School (2016), El gasto farmacéutico en España en 2016. Evolución internacional y situación desde el punto de vista nacional. Disponible en: http://static. correofarmaceutico.com/docs/2016/09/05/gasto-farmaceutico-2016.pdf $[07$ de septiembre de 2017].

Fomento de Estudios Sociales y de Sociología Aplicada (Foessa) (2014), VII Informe sobre exclusión social y desarrollo social en Andalucia y España, España: Fomento de Estudios Sociales y de Sociología Aplicada (Foessa). Disponible en: http://www.foessa2014.es/ informe/uploaded/descargas/VII_INFORME.pdf [23 de septiembre de 2017].

Freire, José Manuel (2014), "Los retos de las reformas sanitarias en el Sistema Nacional de Salud”, en Mediterráneo económico, núm. 26, España: Cajamar.

Garrido, Nuria María (2014), "Hacia una construcción del derecho a la salud como contenido del derecho fundamental a una vida digna y de calidad: el 'mínimo vital' como límite de la sostenibilidad económica”, en Balaguer, Francisco y Arana, Estanislao [comps.], Libro homenaje al profesor Rafael Barranco Vela, España: Thomson ReutersCivitas.

Instituto Nacional de Estadística [INE] (2017), Encuesta de condiciones de vida. Disponible en: https://www.ine.es/dyngs/INEbase/es/operacion.htm?c=Estadistica_C\&cid=1 254736176807\&menu=resultados\&idp=1254735976608 [18 de octubre de 2017].

Jiménez-Martín, Sergi y Viola, Analía Andrea (2014), "El sistema de salud en España en perspectiva comparada”, en Observatorio de la Sanidad FEDEA, España: Fundación de Estudios de Economía Aplicada (FEDEA). Disponible en: http://www.sanidad.fedea. net/docs/ informe.pdf [16 de septiembre de 2017].

Jiménez-Martín, Sergi y Viola, Analía Andrea (2016), "Consumo de medicamentos y copago farmacéutico", en Observatorio de la Sanidad FEDEA, España: Fundación de Estudios de Economía Aplicada (FEDEA). Disponible en: http://www.documentos. fedea.net/pubs/eee/eee2016-06.pdf [16 de septiembre de 2017].

Lago, Juan Aitor et al. (2012), El gasto farmacéutico en España. Evolución internacional, situación nacional y medidas de control del gasto, España: Escuela de Administración de Empresas Business School. Disponible en: htpp://www.redaccionmedica.com/ contenido/images/eae_gastofarmaceutico.pdf [16 de septiembre de 2017].

Landsman, Pamela et al. (2005), "Impact of 3-tier-pharmacy benefit design and increased consumer cost-sharing on drug utilization", en The American Journal of Managed Care, núm. 11, vol. 10, Estados Unidos: The American Journal of Managed Care (AJMC).

Lifshitz, Alberto (2014), "La medicina curativa y la medicina preventiva: alcances y limitaciones”, en Medicina Interna de México, núm. 30, México: Colegio de Medicina Interna de México.

Lucas, Manuel Lucas (2016), "Copago farmacéutico: reflexiones jurídicas en aras a su eventual reforma”, en Nueva fiscalidad, núm. 4, España: Dykinson.

Marshall, Thomas Humphrey (1950), Citizenship and social class, Reino Unido: The syndics of the Cambridge University Press.

Ministerio de Sanidad, Servicios Sociales e Igualdad (2012), Nota de Prensa: Resolución por la que se revisa la lista de medicamentos con financiación pública. Disponible en: http://www.mscbs.gob.es/gabinete/notasPrensa. do?id=2532 [07 de octubre de 2017].

Ministerio de Sanidad, Servicios Sociales e Igualdad (2014-2017), Barómetros Sanitarios (2013-2016). Disponible en: https://www.mscbs.gob.es/estadEstudios/estadisticas/ inforRecopilaciones/barometro/home.htm [20 de noviembre de 2017]. 
Navarro, María Dolores et al. (2016), "Equilibrio entre salud y sanidad", en Torres, Cristóbal [comps.], España 2015, Situación Social, España: Centro de Investigaciones Sociológicas.

Ortún, Vicente (2008), "El impacto de los medicamentos en el bienestar. Informe SESPAS 2008”, en Gaceta Sanitaria, núm. 22, España: Sociedad Española de Salud Pública y Administración Sanitaria.

Oxfam (2016), Una economia al servicio del 1\%, España: Oxfam. Disponible en: http://www.oxfam.org/sites/www.oxfam.org/files/file_attachments/ bp210economyonepercenttax-havens-180116-es_0.pdf [16 de septiembre de 2017].

Páez, Ricardo (2011), "La investigación de la industria farmacéutica: condicionada por los intereses del mercado”, en Acta Bioethica, núm. 11, vol. 2, Chile: Universidad de Chile.

Páez, Ricardo (2016), "La investigación internacional de nuevos medicamentos: una valoración desde la justicia global”, en Revista Latinoamericana de Bioética, núm. 16, vol. 2, Colombia: Universidad Militar Nueva Granada.

Puig-Junoy, Jaume (2007), "La corresponsabilidad individual en la financiación de medicamentos: evidencia y recomendaciones", en Puig-Junoy, Jaume [comp.], La corresponsabilidad individual en la financiación pública de la atención sanitaria, España: Fundación Rafael Campalans. Disponible en: http://www.fcampalans.cat/ publicacions_detall.php?id=8\&idpubli=84 [16 de septiembre de 2017].

Rawls, John (1991), Teoría de la justicia. México: Fondo de Cultura Económica.

Rodríguez, Marisol y Puig-Junoy, Jaume (2013), “Cuando hay que pagar, a veces lo urgente puede esperar”, en Emergencias, núm. 25, vol. 6, España: Revista Científica de la Sociedad Española de Medicina de Urgencias y Emergencias.

Samaja, Juan (2018), "La triangulación metodológica. (Pasos para la comprensión dialéctica de la combinación de métodos)", en Revista Cubana de Salud Pública, núm. 44, vol. 2 , Cuba: Sociedad Cubana de Salud Pública.

Saludas, José Manuel (2013), "Efecto de los copagos en la sanidad: teoría y evidencia”, en Boletín Económico de ICE, núm. 3035, España: Ministerio de Economía y Competitividad. Disponible en: http://www.revistasice.com/es-ES/BICE/Paginas/ Todos-los-Boletines.aspx [22 de diciembre de 2017].

Sanfélix-Gimeno, Gabriel et al. (2011), "La prescripción farmacéutica en Atención Primaria. Mucho más que un problema de gasto", en Ortún, Vicente [comp.], La refundación de la atención primaria, España: Acta Sanitaria. Disponible en: https://link.springer. com/book/10.1007/978-84-938062-5-5 [26 de diciembre de 2017]. 


\section{Anexo}

\section{Tabla I}

\section{Cambios legislativos más relevantes en relación con el copago farmacéutico en España}

\begin{tabular}{|c|c|}
\hline Norma Jurídica & Modificaciones legales más relevantes \\
\hline $\begin{array}{l}\text { Decreto } \\
3157 / 1966 \text {, del } 23 \\
\text { de diciembre }\end{array}$ & $\begin{array}{l}\text { Gratuidad de la asistencia farmacéutica en instituciones sanitarias. } \\
\text { Recoge el copago farmacéutico estableciéndose el límite en las } 30 \text { pesetas } \\
\text { con un copago mínimo de } 5 \text { pesetas }(16,6 \%) \text { y con límite máximo de } 50 \\
\text { pesetas. } \\
\text { No se establecía ningún descuento para ningún colectivo. }\end{array}$ \\
\hline $\begin{array}{l}\text { Real Decreto } \\
945 / 1978 \text {, del } 14 \\
\text { de abril }\end{array}$ & $\begin{array}{l}\text { Establece descuentos en el copago farmacéutico para los pensionistas y las } \\
\text { personas en situación de invalidez. } \\
\text { Establece un copago general de } 30 \% \text { (a excepción de un catálogo de medica- } \\
\text { mentos que se seguirá rigiendo por la norma anterior). }\end{array}$ \\
\hline $\begin{array}{l}\text { Real Decreto } \\
1605 / 1980 \text {, del } \\
31 \text { de julio }\end{array}$ & $\begin{array}{l}\text { Se establece un copago general de } 40 \% \text {. } \\
\text { Se mantiene la excepción de las personas recogidas en la norma anterior. }\end{array}$ \\
\hline $\begin{array}{l}\text { Ley } 29 / 2006 \text {, del } \\
26 \text { de julio }\end{array}$ & $\begin{array}{l}\text { Excluye medicamentos del catálogo de protección farmacéutica (estéticos, } \\
\text { publicitarios, homeopáticos, etcétera). } \\
\text { Se establece una "aportación normal" de } 40 \% \text {. } \\
\text { Se establece una "aportación reducida" donde se abonará el 10\% con un } \\
\text { límite máximo de } 2,64 € \text {. } \\
\text { Ampliación de los colectivos exentos del copago. }\end{array}$ \\
\hline $\begin{array}{l}\text { Real Decreto-ley } \\
\text { 16/2012, del } 20 \\
\text { de abril }\end{array}$ & $\begin{array}{l}\text { El copago estará determinado por la capacidad económica. } \\
\text { Renta igual o superior a } 100.000 € \text { : aportarán } 60 \% \text { del producto. } \\
\text { Renta entre } 18.000-100.00 € \text { : copago será de } 50 \% \text { del producto. } \\
\text { Renta inferior a } 18.000 € \text { : participarán con } 40 \% \text { del producto. } \\
\text { Pensionistas (como norma general): aportarán } 10 \% \text { al producto fijándose } \\
\text { unos límites a su participación: } \\
\text { - Cuando tomen medicamentos pertenecientes al grupo ATC participarán } \\
\text { con un } 10 \% \text { sin que en ningún caso puedan superar los } 2,64 € \text {. } \\
\text { - Quienes tengan unos ingresos por debajo de } 18.000 €: 8 € \text {. } \\
\text { - Cuando los ingresos sean entre } 18.000 € \text { y } 100.000: 18 € . \\
\text { - Si los ingresos son iguales o superiores a } 100.000 €: 60 € \text {. } \\
\text { Se establecen excepciones además de las personas pensionistas, entre ellos, } \\
\text { personas perceptoras de rentas de integración social, de pensiones no contri- } \\
\text { butivas o subsidios por desempleo. No se recoge ninguna excepción para las } \\
\text { personas desempleadas que cobren la prestación por desempleo o no cobren } \\
\text { ningún ingreso. }\end{array}$ \\
\hline
\end{tabular}

Fuente: $\operatorname{BOE}(1966 ; 1978,1980,2006,2012)$, elaboración propia. 


\section{Tabla II}

\section{Sistema de copago farmacéutico}

\begin{tabular}{|c|c|c|}
\hline $\begin{array}{l}\text { CÓDIGO } \\
\text { DE TSI }\end{array}$ & COLECTIVO & $\begin{array}{l}\text { COPAGO } \\
\text { según el PVP }\end{array}$ \\
\hline \multirow{5}{*}{001} & Pensiones no contributivas y sus beneficiarios & \multirow{5}{*}{$0 \%$} \\
\hline & $\begin{array}{l}\text { Parados que han perdido el derecho a percibir el subsidio de desem- } \\
\text { pleo en tanto dure su situación (inscritos en demanda de empleo) }\end{array}$ & \\
\hline & Afectados de síndrome tóxico & \\
\hline & Perceptores de rentas de integración social & \\
\hline & $\begin{array}{l}\text { Tratamientos derivados de accidente de trabajo y enfermedad } \\
\text { profesional }\end{array}$ & \\
\hline \multirow[b]{2}{*}{$002^{* *}$} & $\begin{array}{l}\text { Pensionistas con ingresos inferiores a } 18.000 \text { euros al año, con un } \\
\text { límite mensual de } 8,23 €^{*}\end{array}$ & \multirow[t]{2}{*}{$10 \%$} \\
\hline & $\begin{array}{l}\text { Pensionistas con ingresos de entre 18.000-100.000 euros al año, con } \\
\text { límite mensual de } 18,52 €\end{array}$ & \\
\hline 003 & $\begin{array}{l}\text { Trabajadores activos y sus beneficiarios con ingresos inferiores a } \\
18.000 €\end{array}$ & $40 \%$ \\
\hline 004 & $\begin{array}{l}\text { Trabajadores activos y sus beneficiarios con ingresos de entre } 18.000 \\
\text { y } 100.000 \text { euros al año }\end{array}$ & $50 \%$ \\
\hline \multirow[b]{2}{*}{005} & $\begin{array}{l}\text { Trabajadores activos y sus beneficiarios con ingresos superiores a } \\
100.000 \text { euros }\end{array}$ & \multirow[b]{2}{*}{$60 \%$} \\
\hline & $\begin{array}{l}\text { Pensionistas con ingresos superiores a } 100.000 \text { euros al año, con un } \\
\text { límite mensual de } 61,75 €^{*}\end{array}$ & \\
\hline 006 & $\begin{array}{l}\text { Mutualistas y clases pasivas (funcionarios del Estado, Instituto } \\
\text { Social de las Fuerzas Armadas y la Mutualidad General Judicial) }\end{array}$ & $30 \%$ \\
\hline
\end{tabular}

${ }^{*}$ Estas cantidades se revalorizarán anualmente según las variaciones del IPC. ${ }^{* *}$ En el caso de medicamentos de aportación reducida y grupos ATC abonarán un máximo de 4,24€ por receta.

Fuente: BOE (2015), elaboración propia. 


\section{Gráfico 1}

\section{Evolución del gasto farmacéutico público y personal por habitante en euros}

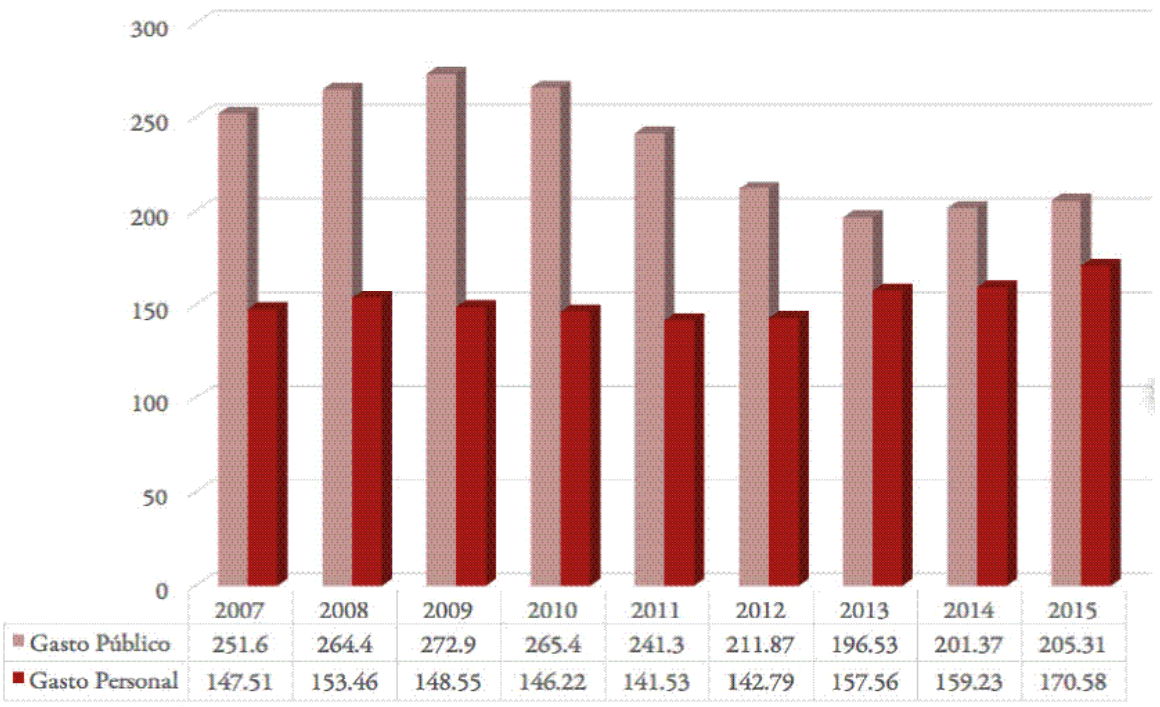

Fuente: EAE Business School (2016), elaboración propia. 


\section{Gráfico 2}

Evolución del porcentaje de personas que no puede acceder a la medicación prescrita en España

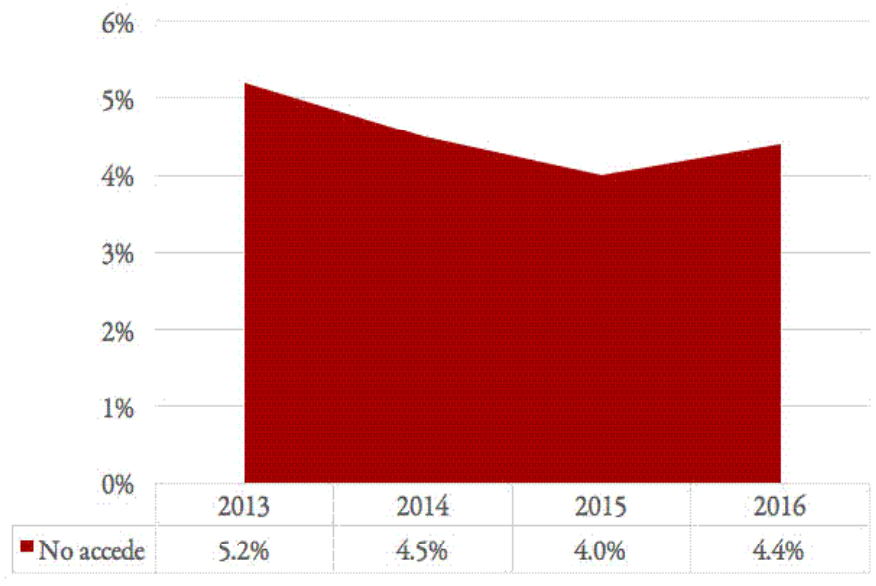

Fuente: Barómetro sanitario del Ministerio de Sanidad, Servicios Sociales e Igualdad (2014-2017), elaboración propia. 
José Ángel Martínez-López. Doctor por la Universidad de Murcia. Profesor Ayudante-Doctor de la Universidad de Murcia, España, Facultad de Trabajo Social, Departamento de Trabajo Social y Servicios Sociales, España. Líneas de investigación: política social, Estado de bienestar, cuidados de larga duración, dependencia y desigualdad social. Publicaciones recientes: MartínezGayo, Gema y Martínez-López, José Ángel, “Copago de medicamentos y debilitación de la ciudadanía social. Una reflexión crítica de los cambios legislativos en materia farmacológica”, en La Razón histórica. Revista Hispanoamericana de Historia de las Ideas, núm. 39, España (2018). Disponible en: https://www.revista larazonhistorica.com/39-6/; Martínez-López, J. A., "El modelo híbrido de atención a las personas en situación de dependencia en España: una década de cambios normativos y ajustes presupuestarios”, en Reforma y Democracia, núm. 68, Venezuela: Centro Latinoamericano de Administración para el Desarrollo (2017); Martínez-López, J. A., Frutos, L., y Solano, J. C., "Los usos de las prestaciones económicas de la dependencia en el municipio de Murcia. Un estudio de caso", en Revista Española de Sociología, núm. 26, España: Federación Española de Sociología (2017).

Gema Martínez-Gayo. Máster Universitario en Problemas Sociales y en la actualidad investigadora en formación (Doctoranda) en el Programa de Doctorado en Análisis de Problemas Sociales de la Universidad Nacional de Educación a Distancia (UNED), España. Líneas de investigación: mercado de trabajo y precariedad laboral, turismo, género, desigualdad social y sinhogarismo. Publicaciones recientes: Martínez-Gayo, Gema y Martínez-López, José Ángel, "Copago de medicamentos y debilitación de la ciudadanía social. Una reflexión crítica de los cambios legislativos en materia farmacológica”, en La Razón Histórica. Revista Hispanoamericana de Historia de las Ideas, núm. 39, España (2018). Disponible en: https://www.revistalarazon historica. com/39-6/; Martínez-Gayo, Gema, "Las personas sin hogar del Municipio de Oviedo: Características y necesidades", en Ayuntamiento de Oviedo, Principado de Asturias, España (2016); Martínez-Gayo, Gema y Martínez Quintana, Violante, "El estatus de los trabajadores extranjeros en el mercado laboral español en un contexto de crisis económica”, en González García, Eduardo et al. [comps.], Mundos emergentes: cambios, conflictos y expectativas, Toledo: Asociación Castellano-Manchega de Sociología, España (2015). Disponible: https://dialnet.unirioja.es/servlet/articulo?codigo $=5532310$. 